\title{
Micrornas as Double Edged Sword in Cancer
}

\author{
${ }^{1}$ Baby Joseph and ${ }^{2}$ Vrundha M. Nair \\ ${ }^{1}$ Department of Biotechnology, \\ ${ }^{2}$ Department of Bioinformatics, \\ Malankara Catholic College, Mariagiri, Kaliakkavilai-629153, \\ Kanyakumari District, TamilNadu, India
}

Received 2010-01-23, Revised 2012-09-19; Accepted 2013-05-24

\begin{abstract}
Cancer is the leading causes of death world wide. Basically it can be defined as the disease of the cell. One among the mechanism of transfer of a normal cell to cancerous is the alteration in the genetic material DNA. Treatment for cancer remains as a biggest challenge. Here comes the importance of miRNAs. They are endogenous RNA which regulates a wide range of cellular processes such as proliferation, differentiation, development and apoptosis by suppressing the expression of target mRNA thus playing a central role in various human diseases including cancer. Hence advancement in miRNA research is necessary to develop it as a powerful therapeutic tool in cancer. Oncomirs are miRNAs acts as double edged sword in cancer because up-regulation and down-regulation of miRNAs are observed in cancerous cells and hence acts as oncogenes and tumor suppressors respectively. miRNAs can act as potential biomarkers. Studies shows that the change in level of miRNA are directly associated with cancer. Hence till-date reviewing as onco-miRs is necessary which further the researcher to develop it as a specific and potential target in cancer treatment. miRNAs are promising therapeutic tools for cancer because in humans, $50 \%$ of miRNA genes were localized in cancer-associated genomic regions which include minimal regions of amplification, loss of heterozygosity, fragile sites and common breakpoint regions. miRNA are found to be up-regulated and down-regulated in almost all cancer cells. Hence, in this article an attempt is made to review the evidence of microRNAs in cancer as both oncogenes and tumor suppressors.
\end{abstract}

Keywords: MicroRNA, Double Edged Sword Tumor Suppressors, Onco-miRs, Gene Regulation

\section{INTRODUCTION}

MicroRNAs are small 22 nucleotides (nt) long, non protein coding, single standed RNAs found in both plants and animals (Bartel, 2004). Lee et al. (1993) in the Victor Ambros lab first discovered miRNA, lin-4 in Caenorhabditis elegans through a genetic screen for defects in the temporal control of post-embryonic development. The term microRNA was coined (Ruvkun, 2001). Unlike siRNA, they are found endogenous (Bartel, 2004; Ambros, 2001; Carrington and Ambros, 2003). Many miRNAs are found to be highly conserved molecules (Pasquinelli et al., 2000). The complete mature miRNA sequence of let-7, isolated primarily in
Caenorhabditis elegans, has been evolutionarily conserved from worms to humans (Lee and Ambros, 2001). Currently, thousands of miRNAs have been identified in nematodes, amphibians, fishes, plants, mammals and viruses (Zhang et al., 2006a) using different approaches including experimental methods, computational approaches, EST and Genomic Survey Sequence analysis (Lee and Ambros, 2001; Brown and Sanseau, 2005; Zhang et al., 2005; 2006b). Out of hundreds of miRNAs that have recently been identified, only about 200-300 miRNAs have been currently identified in humans (Griffiths-Jones, 2004; Griffiths-Jones et al., 2006). Studies prove that miRNAs could also cause gene silencing (Lee et al., 1993; Pasquinelli et al., 2000;

Corresponding Author: Baby Joseph, Department of Biotechnology, Malankara Catholic College, Mariagiri, Kaliakkavilai-629153, Kanyakumari District, Tamil Nadu, India 
Wightman et al., 1993; Olsen and Ambros, 1999; Reinhart et al., 2000). Recent evidence indicates that miRNAs exhibit important regulatory roles in development, cell proliferation, cell survival and apoptosis and thus play a central role in gene regulation in health and disease (Bentwich et al., 2005; He and Hannon, 2004; Meltzer, 2005). In this review, we brief gene regulation of miRNA, its association with cancer, furthermore with instances to prove it as tumor suppressors and oncogenes.

\section{1. miRNA Gene Regulation}

In humans, miRNAs target more than $30 \%$ of protein coding genes (Berezikov et al., 2005; Lewis et al., 2005) and the total number of miRNAs by computational analysis indicates there may be more than $1 \%$ of the total protein coding genes (Lai et al., 2003; Lim et al., 2003a; 2003b). MicroRNA biogenesis and mechanism of gene repression are similar to those of exogenous small interfering RNAs (siRNAs) (Izquierdo, 2005). The genes encoding miRNAs are much longer than the processed mature miRNA molecule. MicroRNAs negatively regulate the target gene expression in a variety of ways including translational repression, mRNA cleavage and deadenylation. MicroRNAs can direct RISC to downregulate gene expression by binding with perfect or nearly perfect complementarity to protein coding mRNA sequences. Most animal miRNAs are thought to use a gene regulation mechanism that does not involve the cleavage of their mRNA targets (Filipowicz et al., 2005). RISC is a ribonucleoprotein complex containing members of the Argonaute (Ago) family of proteins. Argonaut is also partially responsible for selection of the guide strand (incorporated strand) on the basis of the stability of the $5^{\prime}$ end and destruction of the passenger strand. The strand with lower stability base pairing of the 2-4 nt at the 5' end of the duplex preferentially associates with RISC and thus becomes the active miRNA (Schwarz et al., 2003). MiRNA genes can be located in the introns and/or exons of protein-coding genes or in the intergenic regions between protein-coding genes. They can form polycistronic clusters or exist individually (Bartel, 2004; Lagos-Quintana et al., 2001; Kim and Nam, 2006). A single miRNA could regulate multiple target genes, while a single gene could be targeted by multiple miRNA (Wu et al., 2006). Location of miRNA near protein coding genes and its ability to regulate genes proves its importance in medicine.

\section{2. miRNAs and Cancer}

Cancer is a complex genetic disease in which oncogene amplication and/or tumor suppressor gene mutation leads to step-wise deregulation of cell proliferation and apoptosis (Wu et al., 2006). A number of studies reported that specific microRNA signature had been found in each cancer tissue and microRNA based cancer classification is a very effective and potential tool ( $\mathrm{Lu}$ et al., 2005). The involvement of miRNAs in cancers was confirmed through the observation that miRNAs are frequently located in cancer-associated genomic regions, which include minimal regions of amplification, loss of heterozygosity, fragile sites and common breakpoint regions in or near oncogenes or tumor suppressor genes (Calin et al., 2004a). The first report linking miRNAs and cancer involves CLL (B cell lymphocytic leukemia) (Calin et al., 2002). miRNAs show globally lower expression in cancer tissues than in normal tissues (Lu et al., 2005). Abnormalities in miRNA expression have been implicated in several forms of solid tumors such as cervical (Lee et al., 2008), breast (Iorio et al., 2005), colorectal (Cummins et al., 2006), lung (Hayashita et al., 2005) and also in at least two forms of leukemia (Calin et al., 2004a; 2004b). Avissar et al. (2009) used quantitative RT-PCR to study the expression of miR-375 in Head and Neck Squamous Cell Carcinomas (HNSCCs), found higherexpression of this miRNA in tumors of pharyngeal and laryngeal origin suggesting that alterations in miRNA expression are related to cancers and are useful biomarkers in cancers. This instance shows the role of miRNA in cancer and as potent biomarker.

\section{3. miRNAs as Tumor Suppressor}

A variety of miRNAs have been identified that appear to have tumor suppressor functions. A recent study has shown that there is a global down-regulation of miRNA expression in various tumor tissues ( $\mathrm{Lu}$ et al., 2005). B Cell Lymphocytic Leukemia (CLL) is characterized by the deletion of miR-15a and miR-16-1, two clustered miRNAs, within the 13q14.3 locus (Calin et al., 2002). Deletions at this region also occur in approximately $50 \%$ of mantle cell lymphoma, in $16-40 \%$ of multiple myeloma and in $60 \%$ of prostate cancers, suggesting the location of one or more tumor suppressor genes at this locus (Dong et al., 2001). Northern analysis suggested that both miRNAs were down-regulated in the majority of cases (approximately 70\%). A predicted target of these miRNAs is B cell lymphoma $2(\mathrm{Bcl} 2)$, an anti-apoptotic 
protein. The down-regulation of miR-15 and miR-16 leads to an in increase in $\mathrm{Bcl} 2$ expression (Cimmino et al., 2005). miR-15a and miR-16-1 were also expressed at lower levels in pituitary adenomas as compared to normal pituitary tissue and their expression inversely correlated with tumor size (Bottoni et al., 2005).

Let-7 miRNAs are considered as classical tumor suppressors due to their frequent down-regulation in cancers like lung or colon (Lu et al., 2005; Volinia et al., 2006). Ras oncogenes were the first target described to be regulated by the let-7 miRNA family. In both $C$. elegans and human lung cancer cell lines let-7 negatively regulated RAS (Johnson et al., 2005) and it was not expressed in human lung cancer tissues (Johnson et al., 2005; Takamizawa et al., 2004). Furthermore, overexpression of let-7 in A549 lung adenocarcinoma cell line inhibited lung cancer cell growth (Takamizawa et al., 2004).

Recent studies reported that another target of let-7 miRNA family is High mobility group a2 (Hmga2) protein, oncogenic in a variety of tumors, including benign mesenchymal tumors and lung cancers. Chromosomal translocations disrupt the repression of Hmga2 by let-7 miRNA which promotes anchorageindependent growth, a characteristic of oncogenic transformation (Lee and Dutta, 2007; Mayr et al., 2007). In lung cancer cell line restoration of the steady state levels of let-7 inhibited cell replication. Studies in human colon cancer tumors and cell lines show that in addition to Ras, c-Myc might also be a target of let-7 as its expression reduces levels of RAS and c-MYC proteins (Akao et al., 2006). miR-143 and miR-145 has been found to be down-regulated in colorectal cancers (Slaby et al., 2007), B-cell lymphomas (Akao et al., 2007) and in cervical cancers (Lui et al., 2007; Martinez et al., 2008). A recent report showed that TCL1, an oncogene that is overexpressed in CLL cells (Herling et al., 2006) and Mcl-1, an anti-apoptotic Bcl-2 family member (Mott et al., 2007) are targets of miR-29 genes (Pekarsky et al., 2006).

Ciafre et al. (2005) identified a group of miRNAs: $m i R-128, m i R-181 \mathrm{a}, m i R-181 \mathrm{~b}$ and $m i R-181 \mathrm{c}$ were down-regulated in glioblastoma (Ciafre et al., 2005). In primary neuroblastoma tumors, miR-34a on chromosome 1p36.23 was generally expressed at lower levels and it directly targeted the mRNA encoding E2F3 and significantly reduced E2F3 protein levels. This result suggested that miR-34a acted as a tumor suppressor of neuroblastoma tumorigenesis (Welch et al., 2007). MiR34a was frequently absent in pancreatic cancer cells and its responsive genes were highly enriched for those that regulated cell-cycle progression, apoptosis, DNA repair and angiogenesis (Chang et al., 2007). Slack's research group demonstrates that let-7 miRNA inhibits the growth of lung cancer cells in culture and in lung tumors in mice. They also showed that let-7 can be applied as an intranasal drug to reduce tumor formation in a RAS mouse model lung cancer (Esquela-Kerscher et al., 2008).

Bhattacharya et al. (2009) identified two miRNAs, miR-15a and miR-16, that are underexpressed in ovarian cell lines and in primary ovarian tissues. Oncogenic activation of Bmi-1 is found in a wide variety of epithelial malignancies including ovarian cancer. Bmi-1 protein levels are down-regulated in response to miR-15a or miR-16 expression and lead to significant reduction in ovarian cancer cell proliferation and clonal growth, suggesting the development of therapeutic strategies by restoring miR-15a and miR-16 expression in ovarian cancer and in other cancersn (Bhattacharya et al., 2009).

\section{4. miRNA as oncogenes}

He and colleagues found the over expression of the miR-17-92 polycistron at $13 \mathrm{q} 31.3$ in B-cell lymphomas (He et al., 2005). It is also found to be over expressed in several other cancers such as solid cancers (Volinia et al., 2006), lung cancer (Hayashita et al., 2005) and malignant lymphoma cell lines (Tagawa and Seto, 2005) proving it as potential oncogenes. Recent studies in mouse B-cell lymphoma model reveals miR-19 is a key oncogenic componenet of mir-17-92 cluster (Olive et al., 2009). In breast tumors $m i R-21$ was found to be over expressed when compared to normal breast tissues (Iorio et al., 2005; Si et al., 2007). Zhu et al. (2007) identified down-regulation of tumor suppressor, tropomyosin 1 in breast cancer by $m i R-21$ could result in tumor growth supporting the notion that tropomyosin 1 as a potential $m i R-21$ target. It was found to be over expressed in head and neck cancer cell lines (Tran et al., 2007), brain tumor and glioblastoma (Chan et al., 2005) proving it as an oncogene. The glioblastoma tissues and glioblastoma cell lines analysis shows strong upregulation of miR-221 (Ciafre et al., 2005).

In Cervical cancer tissues, increased expression of miR-15b, miR-16, miR-146a, miR-155 and miR-223 has been observed. Cimmino et al. (2005) reported that miR15 and miR-16 regulate apoptosis by targeting BCL2. miR-155 was found over expressed in lung cancer (Yanaihara et al., 2006), lymphoblastic leukemia/highgrade lymphoma (Calin et al., 2005), B-cell 
lymphomas, Hodgkin's lymphomas, Burkitt lymphomas and in human breast cancer cells suggesting that it may act as oncogene (Iorio et al., 2005; Metzler et al., 2004; Eis et al., 2005; Kluiver et al., 2005).

Studies proved that miRNA expression can be regulated by DNA methylation and it has been suggested that altered miRNA gene methylation might contribute to human tumorigenesis. Let-7a-3 was found to be methylated by the DNA methyltransferases DNMT1 and DNMT3B. The gene was heavily methylated in normal human tissues but hypomethylated in some lung adenocarcinomas. Brueckner et al. (2007) identified let- 7a-3 as epigenetically regulated miRNA gene with oncogeneic function and suggest that aberrant miRNA gene methylation might contribute to the human cancer epigenome. Analysis of human breast tumors by Wang et al. (2009) revealed that miR-27b expression increases during cancer progression, paralleling a decrease in Suppressor of Tumorigenicity 14 (ST14) expression. The 3 '-untranslated region of ST14 contains a regulatory element for miR-27b and luciferase experiments indicate that antisense miR-27b enhances ST14 expression in cancer cells which reduces cell proliferation as well as cell migration and invasion. Knockdown or over expression of a specific miRNA allows studying the specific roles of the miRNA in cancer development.

\section{CONCLUSION}

Oncogenic micro RNAs or Oncomirs are miRNAs with a role in cancer. The first functional evidence of a miRNA, or any non-coding RNA, acting as a mammalian oncogene is about mir-17-92 from studies of Hammond (2006) in B-cell lymphoma. For this reason they refer the host transcript of mir-17-92 as OncomiR-1 (Hammond, 2006). The above discussions clearly reveal microRNas can act both as tumor suppressors and oncogenes. They can be considered as potential therapeutic targets for cancer. Antisense inhibitors could be used to target oncogenic miRNAs and desired therapeutic strategy would increase the function of tumor suppressor miRNAs in cells. The small size and molecular properties of miRNAs makes it agreeable targets and therapeutics in cancer treatment.

\subsection{Future Prospective}

All non coding RNAs are not miRNAs, hence identifying a common sequence signature or biochemical action helps to overcome this challenge. Little is known about how miRNAs are regulated; much less what polymerase transcribes them. Furthermore, nothing is known about what signals conveys the temporal and/or spatial expression of miRNAs. This can be predicted to become an active area of research that will be highly important in the study of development and disease. Moreover predicting the impact of miRNAs on target proteins is challenging because of their different regulatory effects at the transcriptional and translational levels. Using chromatin modifying drugs to activate tumor suppressor miRNAs can regulate target oncogenes and it may lead to novel cancer therapies in the future. miRNAs can complement other genomic and proteomic biomarkers for cancer diagnosis and prognosis (Cho, 2007; Cho and Cheng, 2007). While hundreds of human microRNAs are known, relatively little is known about their roles and targets. Effective delivery of microRNA in to targeted tissues and maintaining their continuous activity still remains as an obstacle. Once overcoming all these difficulties miRNA remains as a promising cancer therapeutic tool.

\section{REFERENCES}

Akao, Y., Y. Nakagawa and T. Naoe, 2006. Let-7 microrna functions as a potential growth suppressor in human colon cancer cells. Biol. Pharm. Bull., 29: 903-906. DOI: 10.1248/bpb.29.903

Akao, Y., Y. Nakagawa, Y. Kitade, T. Kinoshita and T. Naoe, 2007. Downregulation of microRNAs-143 and 145 in B-cell malignancies. Cancer Sci., 98: 19141920. DOI: 10.1111/j.1349-7006.2007.00618.x

Ambros, V., 2001. MicroRNAs: Tiny regulators with great potential. Cell, 107: 823-826. DOI: 10.1016/S0092-8674(01)00616-X

Avissar, M., M.D. McClean, K.T. Kelsey and C.J. Marsit, 2009. MicroRNA expression in head and neck cancer associates with alcohol consumption and survival. Carcinogenesis, 30: 2059-2063. DOI: 10.1093/carcin/bgp277

Bartel, D.P., 2004. MicroRNAs: Genomics, biogenesis, mechanism and function. Cell, 116: 281-297. DOI: 10.1016/S0092-8674(04)00045-5

Bentwich, I., A. Avniel, Y. Karov, R. Aharonov and S. Gilad et al., 2005. Identification of hundreds of conserved and nonconserved human microRNAs. Nat. Genet., 37: 766-770. DOI: 10.1038/ng1590

Berezikov, E., V. Guryev, J.V.D. Belt, E. Wienholds, and E. Cuppen et al., 2005. Phylogenetic shadowing and computational identification of human microRNA genes. Cell, 120: 21-24. DOI: 10.1016/j.cell.2004.12.031 
Bhattacharya, R., M. Nicoloso, R. Arvizo, E. Wang and A. Cortez et al., 2009. Mir-15a and MiR-16 control Bmi-1 expression in ovarian cancer. Cancer Res., 69: 9090-9095. DOI: 10.1158/0008-5472.CAN-09-2552

Bottoni, A., D. Piccin, F. Tagliati, A. Luchin and M.C. Zatelli et al., 2005. Mir-15a and miR-16-1 downregulation in pituitary adenomas. J. Cell. Physiol., 204: 280-285. DOI: 10.1002/jcp.20282

Brown, J.R. and P. Sanseau, 2005. A computational view of microRNAs and their targets. Drug Discov. Today, 10: 595-601. DOI: 10.1016/S1359-6446(05)03399-4

Brueckner, B., C. Stresemann, R. Kuner, C. Mund and T. Musch et al., 2007. The human let-7a-3 locus contains an epigenetically regulated microRNA gene with oncogenic function. Cancer Res., 67: 14191423. DOI: 10.1158/0008-5472.CAN-06-4074

Calin, G.A., C. Sevignani, C.D. Dumitru, T. Hyslop and E. Noch et al., 2004a. Human microRNA genes are frequently located at fragile sites and genomic regions involved in cancers. PNAS, 101: 2999-3004. DOI: $10.1073 /$ pnas.0307323101

Calin, G.A., C.D. Dumitru, M. Shimizu, R. Bichi and S. Zupo et al., 2002. Frequent deletions and downregulation of micro- RNA genes miR15 and miR16 at 13 q14 in chronic lymphocytic leukemia. Proc. Natl. Acad. Sci., 99: 15524-15529. DOI: 10.1073/pnas.242606799

Calin, G.A., C.G. Liu, C. Sevignani, M. Ferracin and N. Felli et al., 2004b. MicroRNA profiling reveals distinct signatures in B cell chronic lymphocytic leukemias. Proc. Natl. Acad. Sci. U.S.A., 101: 175511760. DOI: 10.1073/pnas.0404432101

Calin, G.A., M. Ferracin, A. Cimmino, G. di Leva and M. Shimizu et al., 2005. A MicroRNA signature associated with prognosis and progression in chronic lymphocytic leukemia. New Engl. J. Med., 353: 1793-1801. PMID: 16251535

Carrington, J.C. and V. Ambros, 2003. Role of microRNAs in plant and animal development. Science, 301: 336338. DOI: $10.1126 /$ science. 1085242

Chan, J.A., A.M. Krichevsky and K.S. Kosik, 2005. MicroRNA-21 is an antiapoptotic factor in human glioblastoma cells. Cancer Res., 65: 6029-6033. PMID: 16024602

Chang, T.C., E.A. Wentzel, O.A. Kent, K. Ramachandran and M. Mullendore et al., 2007. Transactivation of miR-34a by 53 broadly influences gene expression and promotes apoptosis. Mol. Cell, 26: 745-752. DOI: 10.1016/j.molcel.2007.05.010
Cho, W.C. and C.H. Cheng, 2007. Oncoproteomics: Current trends and future perspectives. Exp. Rev. Proteomics, 4: 401-410. DOI: 10.1586/14789450.4.3.401

Cho, W.C., 2007. Contribution of oncoproteomics to cancer biomarker discovery. Mol. Cancer, 6: 25-25. DOI: 10.1186/1476-4598-6-25

Ciafre, S.A., S. Galardi, A. Mangiola, M. Ferracin and C.G. Liu et al., 2005. Extensive modulation of a set of microRNAs in primary glioblastoma. Biochem. Biophys. Res. Commun., 334: 1351-1358. DOI: 10.1016/j.bbrc.2005.07.030

Cimmino, A., G.A. Calin, M. Fabbri, M.V. Iorio and M. Ferracin et al., 2005. miR-15 and miR-16 induce apoptosis by targeting BCL2. PNAS, 102: 1394413949. DOI: $10.1073 /$ pnas.0506654102

Cummins, J.M., Y. He, R.J. Leary, R. Pagliarini and L.A. Diaz et al., 2006. The colorectal microRNAome. Proc. Natl. Acad. Sci. U.S.A., 103: 3687-3692. DOI: 10.1073/pnas.0511155103

Dong, J.T., J.C. Boyd and H.F. Frierson Jr., 2001. Loss of heterozygosity at $13 q 14$ and $13 q 21$ in high grade, high stage prostate cancer. Prostate, 49: 166-171. DOI: $10.1002 /$ pros. 1131

Eis, P.S., W. Tam, L. Sun, A. Chadburn and Z. Li et al., 2005. Accumulation of miR-155 and BIC RNA in human B cell lymphomas. Proc. Natl. Acad. Sci., 102: 3627-3632. DOI: 10.1073/pnas.0500613102

Esquela-Kerscher, A., P. Trang, J.F. Wiggins, A. Patrawala and L. Cheng et al., 2008. The let-7 microRNA reduces tumor growth in mouse models of lung cancer. Cell Cycle, 7: 759-64. PMID: 18344688

Filipowicz, W., L. Jaskiewicz, F.A. Kolb and R.S. Pillai, 2005. Post-transcriptional gene silencing by siRNAs and miRNAs. Curr. Opin. Struct. Biol., 15: 331-341. DOI: 10.1016/j.sbi.2005.05.006

Griffiths-Jones, S., 2004. The microRNA registry. Nucl. Acids Res., 32: 109-111. DOI: 10.1093/nar/gkh023

Griffiths-Jones, S., R.J. Grocock, S.V. Dongen, A. Bateman and A.J. Enright, 2006. Mirbase: MicroRNA sequences, targets and gene nomenclature. Nucl. Acids Res., 34: 140-144. DOI: 10.1093/nar/gkj112

Hammond, S.M., 2006. MicroRNAs as oncogenes. Curr. Opin. Genet. Dev., 16: 4-9. PMID: 16361094

Hayashita, Y., H. Osada, Y. Tatematsu, H. Yamada and K. Yanagisawa et al., 2005. A polycistronic microRNA cluster, miR-17-92, is overexpressed in human lung cancers and enhances cell proliferation. Cancer Res., 65: 9628-9632. PMID: 16266980 
He, L. and G.J. Hannon, 2004. MicroRNAs: Small RNAs with a big role in gene regulation. Nat. Rev. Genet., 5: 522-531. DOI: 10.1038/nrg1379

He, L., J.M. Thomson, M.T. Hemann, E. HernandoMonge and $\mathrm{D}$. $\mathrm{Mu}$ et al., 2005. A microRNA polycistron as a potential human oncogene. Nature, 435: 828-833. DOI: 10.1038/nature03552

Herling, M., K.A. Patel, J. Khalili, E. Schlette and R. Kobayashi et al., 2006. TCL1 shows a regulated expression pattern in chronic lymphocytic leukemia that correlates with molecular subtypes and proliferative state. Leukemia, 20: 280-285. DOI: 10.1038/sj.leu.2404017

Iorio, M.V., M. Ferracin, C.G. Liu, R. Veronese and A. Spizzo et al., 2005. MicroRNA gene expression deregulation in human breast cancer. Cancer Res., 65: 7065-7070. PMID: 16103053

Izquierdo, M., 2005. Short interfering RNAs as a tool for cancer gene therapy. Cancer Gene Ther., 12: 217227. DOI: $10.1038 /$ sj.cgt.7700791

Johnson, S.M., H. Grosshans, J. Shingara, M. Byrom and R. Jarvis et al., 2005. RAS is regulated by the let-7 microRNA family. Cell, 120: 635-647. DOI: 10.1016/j.cell.2005.01.014

Kim, V.N. and J.W. Nam, 2006. Genomics of microRNA. Trends Genet, 22: 165-173. DOI: 10.1016/j.tig.2006.01.003

Kluiver, J., S. Poppema, D. Jong, T. Blokzijl and G. Harms et al., 2005. BIC and miR-155 are highly expressed in Hodgkin, primary mediastinal and diffuse large B cell lymphomas. J. Pathol., 207: 243249. DOI: $10.1002 /$ path. 1825

Lagos-Quintana, M., R. Rauhut and W. Lendeckel, 2001. Identification of novel genes coding for small expressed RNAs. Science, 294: 853-858. DOI: 10.1126/science. 1064921

Lai, E.C., P. Tomancak, R.W. Williams and G.M. Rubin, 2003. Computational identification of Drosophila microRNA genes. Genome Biol., 4: R42-R42. PMID: 12844358

Lee, J.W., C.H. Choi, J.J. Choi, Y.A. Park and S.J. Kim et al., 2008. Altered microrna expression in cervical carcinomas. Clin. Cancer Res., 14: 2535-2542. DOI: 10.1158/1078-0432.CCR-07-1231

Lee, R.C. and V. Ambros, 2001. An extensive class of small RNAs in Caenorhabditis elegans. Science, 294: 862-864. DOI: 10.1126/science.1065329

Lee, R.C., R.L. Feinbaum and V. Ambros, 1993. The $C$. elegans heterochronic gene lin-4 encodes small RNAs with antisense complementarity to lin-14. Cell, 75: 843-854. DOI: 10.1016/0092-8674(93)90529-Y
Lee, Y.S. and A. Dutta, 2007. The tumor suppressor microRNA let-7 represses the HMGA2 oncogene. Genes Dev., 21: 1025-1030. DOI: 10.1101/gad.1540407

Lewis, B.P., C.B. Burge and D.P. Bartel, 2005. Conserved seed pairing, often flanked by adenosines, indicates that thousands of human genes are microRNA targets. Cell, 120: 15-20. DOI: 10.1016/j.cell.2004.12.035

Lim, L.P., M.E. Glasner, S. Yekta, C.B. Burge and D.P. Bartel, 2003a. Vertebrate microRNA genes. Science, 299: 1540-1540. DOI: 10.1126/science. 1080372

Lim, L.P., N.C. Lau, E.G. Weinstein, A. Abdelhakim and S. Yekta et al., 2003b. The microRNAs of Caenorhabditis elegans. Genes Dev., 17: 991-1008. DOI: $10.1101 /$ gad.1074403

Lu, J., G. Getz, E.A. Miska, E. Alvarez-Saavedra, J. Lamb and D. Peck et al., 2005. MicroRNA expression profiles classify human cancers. Nature, 435: 834-838. DOI: 10.1038/nature03702

Lui, W.O., N. Pourmand, B.K. Patterson and A. Fire, 2007. Patterns of known and novel small RNAs in human cervical cancer. Cancer Res., 67: 6031-6043. DOI: 10.1158/0008-5472.CAN-06-0561

Martinez, I., A.S. Gardiner, K.F. Board, F.A. Monzon and R.P. Edwards et al., 2008. Human papillomavirus type 16 reduces the expression of microRNA-218 in cervical carcinoma cells. Oncogene, 27: 2575-2582. DOI: 10.1038/sj.onc. 1210919

Mayr, C., M.T. Hemann and D.P. Bartel, 2007. Disrupting the pairing between let-7 and Hmga2 enhances oncogenic transformation. Science, 315: 1576-1579. DOI: 10.1126/science.1137999

Meltzer, P.S., 2005. Cancer genomics: Small RNAs with big impacts. Nature, 435: 745-746. DOI: $10.1038 / 435745 \mathrm{a}$

Metzler, M., M. Wilda, K. Busch, S. Viehmann and A. Borkhardt, 2004. High expression of precursor microRNA-155/BIC RNA in children with Burkitt lymphoma. Gene Chromosomes Cancer, 39: 167169. DOI: $10.1002 /$ gcc. 10316

Mott, J.L., S. Kobayashi, S.F. Bronk and G.J. Gores, 2007. Mir-29 regulates Mcl-1 protein expression and apoptosis. Oncogene, 26: 6133-6140. DOI: 10.1038/sj.onc. 1210436

Olive, V., M.J. Bennett, J.C. Walker, C. Ma and I. Jiang et al., 2009. Mir-19 is a key oncogenic component of mir-17-92. Genes Dev., 23: 28392849. DOI: $10.1101 /$ gad.1861409 
Olsen, P.H and V. Ambros, 1999. The lin-4 regulatory RNA controls developmental timing in Caenorhabditis elegans by blocking LIN-14 protein synthesis after the initiation of translation. Dev. Biol., 216: 671-680. DOI: 10.1006/dbio.1999.9523

Pasquinelli, A.E., B.J. Reinhart, F. Slack, M.Q. Martindale and M.I. Kuroda et al., 2000. Conservation of the sequence and temporal expression of let-7 heterochronic regulatory RNA. Nature, 408: 86-89. DOI: 10.1038/35040556

Pekarsky, Y., U. Santanam, A. Cimmino, A. Palamarchuk and A. Efanov et al., 2006. Tcl1 expression in chronic lymphocytic leukemia is regulated by $m i R-29$ and miR-181. Cancer Res., 66: 11590-11593. DOI: 10.1158/0008-5472.CAN-06-3613

Reinhart, B.J., F.J. Slack, M. Basson, A.E. Pasquinelli and J.C. Bettinger et al., 2000. The 21-nucleotide let-7 RNA regulates developmental timing in Caenorhabditis elegans. Nature, 403: 901-906. DOI: $10.1038 / 35002607$

Ruvkun, G., 2001. Molecular biology: Glimpses of a tiny RNA world. Science, 294: 797-799. DOI: 10.1126/science. 1066315

Schwarz, D.S., G. Hutvagner, T. Du, Z. Xu and N. Aronin et al., 2003. Asymmetry in the assembly of the RNAi enzyme complex. Cell, 115: 199-208. DOI: 10.1016/S0092-8674(03)00759-1

Si, M.L., S. Zhu, H. Wu, Z. Lu, F. Wu and Y.Y. Mo, 2007. Mir-21-mediated tumor growth. Oncogene, 26: 2799-2803. DOI: 10.1038/sj.onc. 1210083

Slaby, O., M. Svaboda, P. Fabian, T. Smerdova and D. Knoflickova et al., 2007. Altered expression of $m i R-$ 21, miR-31, miR-143 and miR-145 is related to clinicopathologic features of colorectal cancer. Oncology, 72: 397-402. DOI: 10.1159/000113489

Tagawa, H. and M. Seto, 2005. A microRNA cluster as a target of genomic amplification in malignant lymphoma. Leukemia, 19: 2013-2016. DOI: 10.1038/sj.leu.2403942

Takamizawa, J., H. Konishi, K. Yanagisawa, S. Tomida and H. Osada et al., 2004. Reduced expression of the let-7 microRNAs in human lung cancers in association with shortened postoperative survival. Cancer Res., 64: 3753-3756. PMID: 15172979

Tran, N., T. McLean, X. Zhang, C.J. Zhao and J.M. Thomson et al., 2007. MicroRNA expression profiles in head and neck cancer cell lines. Biochem. Biophys. Res. Commun., 358: 12-17. DOI: 10.1016/j.bbrc.2007.03.201
Volinia, S., G.A. Calin, C.G. Liu, S. Ambros and A. Cimmino et al., 2006. A microRNA expression signature of human solid tumors defines cancer gene targets. Proc. Natl. Acad. Sci. U.S.A., 103: $2257-$ 2261. DOI: $10.1073 /$ pnas.0510565103

Wang, Y., R. Rathinam, A. Walch and S.K. Alahari, 2009. ST14 (suppression of tumorigenicity 14) gene is a Target for miR-27b and the inhibitory effect of ST14 on cell growth is independent of miR-27b regulation. J. Biol. Chem., 284: 23094-23106. DOI: 10.1074/jbc.M109.012617

Welch, C., Y. Chen and R.L. Stallings, 2007. MicroRNA-34a functions as a potential tumor suppressor by inducing apoptosis in neuroblastoma cells. Oncogene, 26: 5017-5022. DOI: 10.1038/sj.onc. 1210293

Wightman, B., I. Ha and G. Ruvkun, 1993. Posttranscriptional regulation of the heterochronic gene lin-14 by lin-4 mediates temporal pattern formation in $C$. elegans. Cell, 75: 855-862. PMID: 8252622

Wu, W., M. Sun, G.M. Zou and J. Chen, 2006. MicroRNA and cancer: Current status and prospective. Int. J. Cancer, 120: 953-960. DOI: $10.1002 /$ ijc. 22454

Yanaihara, N., N. Caplen, E. Bowman, M. Seike and K. Kumamoto et al., 2006. Unique microRNA molecular profiles in lung cancer diagnosis and prognosis. Cancer Cell, 9: 189-198. DOI: 10.1016/j.ccr.2006.01.025

Zhang, B., X. Pan, G.P. Cobb and T.A. Anderson, 2006a. Plant microRNA: A small regulatory molecule with big impact. Dev. Biol., 289: 3-16. DOI: 10.1016/j.ydbio.2005.10.036

Zhang, B.H., X.P. Pan and T.A. Anderson, 2006b. Identification of 188 conserved maize microRNAs and their targets. FEBS Lett., 580: 3753-3762. DOI: 10.1016/j.febslet.2006.05.063

Zhang, B.H., X.P. Pan, Q.L. Wang, G.P. Cobb and T.A. Anderson, 2005. Identification and characterization of new plant microRNAs using EST analysis. Cell Res., 15: 336-360. PMID: 15916721

Zhu, S., M.L. Si, H. Wu and Y.Y. Mo, 2007. MicroRNA-21 Targets the tumor suppressor gene Tropomyosin 1 (TPM1). J. Biol. Chem., 282: 14328-14336. DOI: 10.1074/jbc.M611393200 